Research Article

\title{
Pharmacokinetic Comparisons of Naringenin and Naringenin-Nicotinamide Cocrystal in Rats by LC-MS/MS
}

\author{
Dan Xu, Gui-Qiu Zhang, Ting-Ting Zhang, Bo Jin $(\mathbb{D}$, and Chen Ma $\mathbb{1}$ \\ Institute of Materia Medica, Chinese Academy of Medical Sciences and Peking Union Medical College, Beijing 100050, China \\ Correspondence should be addressed to Chen Ma; mach@imm.ac.cn
}

Received 12 November 2019; Revised 19 January 2020; Accepted 17 February 2020; Published 1 April 2020

Academic Editor: Constantinos Georgiou

Copyright ( 92020 Dan Xu et al. This is an open access article distributed under the Creative Commons Attribution License, which permits unrestricted use, distribution, and reproduction in any medium, provided the original work is properly cited.

\begin{abstract}
Naringenin (NAR), 4',5,7-trihydroxydihydroflavone, has a wide range of pharmacological activities but shows poor water solubility and low bioavailability. The pharmacokinetics and bioavailability of naringenin-nicotinamide cocrystal (NAR-NCT), which offers improved solubility, were evaluated in this study. Rats were orally administered NAR, a physical mixture of naringenin and nicotinamide (NAR + NCT), and NAR-NCT. The relative bioavailability of NAR-NCT was $175.09 \%$ of NAR, $\mathrm{C}_{\max }$ was 8.43 and 2.06 times of NAR and NAR + NCT, respectively, Tmax was advanced from $0.49 \mathrm{~h}$ to $0.09 \mathrm{~h}$, CL was decreased from $91.1 \mathrm{~L} / \mathrm{h} / \mathrm{kg}$ to $49.1 \mathrm{~L} / \mathrm{h} / \mathrm{kg}$, and $t_{1 / 2}$ was increased from $5.37 \mathrm{~h}$ to $8.24 \mathrm{~h}$, highlighting its rapid absorption and slow elimination. This study showed that NAR-NCT could improve the bioavailability of NAR.
\end{abstract}

\section{Introduction}

Naringenin (NAR), 4',5,7-trihydroxydihydroflavone (Figure 1), is a dihydroflavonoid widely found in grapefruit, grapes, and citrus fruits of the Rutaceae family. It is of great interest because of its rich pharmacological activity including antioxidation $[1,2]$, antitumour $[3,4]$, anti-inflammatory $[5,6]$, antiradiation $[7,8]$, and immunoregulatory effects [9] as well as having effects on cough and phlegm [10], which has resulted in its wide use in the medicine [11], food, and cosmetics industries. Although it has a variety of pharmacological activities, studies have shown that NAR is poorly soluble in water [12] and has low bioavailability in vivo $[13,14]$.

Cocrystal is defined as the crystal of combination with active pharmaceutical ingredient and cocrystal former. It with specific physical and chemical properties can form through weak noncovalent interactions, for example, hydrogen bonding [15]. Compared with the parent compound, active pharmaceutical ingredients in the form of cocrystal can improve different properties [16], such as altered stability [17, 18], solubility [19], and bioavailability [20, 21]. Previously, we prepared naringenin-nicotinamide cocrystal (NAR-NCT) to improve the solubility of NAR in different
pH buffer solutions [22]. Pharmacokinetic comparisons of NAR and NAR-NCT after oral administration in rats were investigated in this work. After oral administration of NAR, the physical mixture (NAR + NCT), and NAR-NCT, and the plasma concentrations of NAR were determined by LC-MS/ MS. NAR-NCT can improve the bioavailability of NAR and result in faster absorption and slower elimination.

\section{Experimental}

2.1. Materials. Naringenin ( $>96 \%)$ and nicotinamide $(>98 \%$, NCT) were provided by Bailingwei Technology (Beijing, China). Internal standard (IS), hesperetin ( $>98 \%$, Figure 1), was provided from YIFEI (Shanghai, China). Acetic acid was provided from TEDIA (Fairfield, OH, USA). Methanol (HPLC grade), ethyl acetate (GC grade), and other chemicals were provided from ANPEL (Shanghai, China).

2.2. Preparation and Characterization of NAR-NCT. The cocrystal was prepared by solvent evaporation. $50 \mathrm{mg}$ of NAR and $45 \mathrm{mg}$ of NCT (at a molar ratio of NAR and NCT of $1: 2$ ) were mixed with ethyl acetate $(10 \mathrm{~mL})$. After ultrasonic dissolution, the mixtures were heated at $40^{\circ} \mathrm{C}$ for 4 
<smiles>O=C1CC(c2ccc(O)cc2)Oc2cc(O)cc(O)c21</smiles>

(a)<smiles>COc1ccc(C2CC(=O)c3c(O)cc(O)cc3O2)cc1O</smiles>

(b)

FIGURE 1: Chemical structure: (a) naringenin and (b) hesperetin.

hours [22]. Differential scanning calorimetry (DSC) was performed on a DSC-TGA-STARe thermal analyzer (Mettler, Toledo, Switzerland). X-Ray powder diffraction (XRPD) was used to analyse the changes of NAR-NCT. XRPD was conducted with a D8 ADVANCE powder diffractometer (Bruker, Karlsruhe, Germany). Infrared spectroscopy (IR) was performed on a Spectrometer 400 Fourier-infrared spectrometer (PerkinElmer, Waltham, America). The NARNCT was kept at $60^{\circ} \mathrm{C}$, at a relative humidity of $90 \pm 5 \%$, under light of $5000 \mathrm{Lx}$ for 10 days and after 6 months at room temperature.

2.3. Instrumentation. The chromatographic separation was carried out by a Shimadzu UFLC-20AD XR system (Tokyo, Japan). The column was Shim-Pack C18-ODS column $(75 \mathrm{~mm} \times 3 \mathrm{~mm}, 2.3 \mu \mathrm{m})$ at $40^{\circ} \mathrm{C}$ and $0.3 \mathrm{~mL} / \mathrm{min}$. The mobile phase constituted $0.2 \%$ acetic acid-water $(\mathrm{v} / \mathrm{v}, \mathrm{A})$ and methanol (B). The injection volume was $3 \mu \mathrm{L}$. The chromatographic program was as follows: gradient elution, 0-3 min, 60\% B; 3-3.5 min, 60-90\% B; 3.5-6.5 min, 90\% B; 6.5-7 min, 90-60\% B; and 7-10 min, 60\% B.

AB SCIEX Qtrap 5500 (MDS-Sciex, Concord, Canada) with a negative mode electrospray ionization (ESI) interface was equipped with Shimadzu UFLC-20AD XR. Multiple reaction monitoring was used to analyse samples. Mass spectrometry parameters are shown in Table 1 .

Secondary mass spectrometry enhanced product ion (EPI) scan was used to obtain a high-quality MS/MS spectrum on a specific ion. This scan type was performed on NAR and IS. The product ion mass spectra about NAR and IS are recorded in Figure 2. The ratio of peak area about NAR to IS was used to determine concentrations of NAR. Data acquisition and analysis were calculated by Analyst 1.6 software. After that, to minimize the contamination of the sample in the mass spectrometer, after the peaks of NAR and IS had eluted, the remaining components were sent from the LC to the waste valve instead of proceeding through the MS system.

2.4. Standard Working Solutions and Internal Standard Solution. NAR was dissolved in methanol to obtain a $102.6 \mu \mathrm{g} / \mathrm{mL}$ stock solution. It was diluted with methanol to obtain standard working solutions of 10.3, 30.8, 102.6, 513, 1026, 2052, 4104, 6156, and $8208 \mathrm{ng} / \mathrm{mL}$. Hesperidin was dissolved in methanol $(300 \mathrm{ng} / \mathrm{mL})$ as an internal standard solution.
2.5. Calibration Standards and Quality Control (QC) Samples. The calibration standards of NAR at a range of 1.03 to $821 \mathrm{ng} / \mathrm{mL}$, which were prepared from blank plasma and NAR standard working solutions.

QC samples were formulated at low, medium, and high concentrations (LQC, MQC, and HQC) corresponding to NAR of 3.08, 410, and $616 \mathrm{ng} / \mathrm{mL}$, respectively, following the procedure described above. The samples were protected from light.

2.6. Pretreatment of the Plasma Samples. Plasma $(100 \mu \mathrm{L})$ was combined with $10 \mu \mathrm{L}$ of $300 \mathrm{ng} / \mathrm{mL}$ internal standard solution in a centrifuge tube, and the mixture was vortexed for $5 \mathrm{~min}$. Then, samples were mixed with ethyl acetate $(300 \mu \mathrm{L})$, and mixtures were vortex-mixed for $5 \mathrm{~min}$. After that, mixtures were centrifuged at $8000 \mathrm{r} / \mathrm{min}$ for $2 \mathrm{~min}$. All supernatants were pipetted into a new tube and dried at $40^{\circ} \mathrm{C}$ under flowing nitrogen. And then, residues were reconstituted with methanol $(100 \mu \mathrm{L})$ and vortex-mixed for $1 \mathrm{~min}$.

2.7. Method Validations. All method validations complied with US Food and Drug Administration guidelines [23].

2.7.1. Specificity. To demonstrate that substances being measured were intended analytes and endogenous substances and other metabolites do not interfere with the assay, method specificity was established firstly. This analysis involved blank plasma samples (different sources), the mixture of NAR standard solution and blank plasma, and plasma samples after oral administration $(n=6)$.

2.7.2. The Calibration Curve and Lower Limit of Quantification (LLOQ). Six blank rat plasma samples were combined with the NAR standard working solutions to give final NAR plasma concentrations of $1.03,10.3,51.3,103,205$, and $821 \mathrm{ng} / \mathrm{mL}$. As described in Section 2.6, the NAR plasma concentration $(\mathrm{ng} / \mathrm{mL})$ was taken as the abscissa, and the ratio of the NAR to IS peak areas was taken as the ordinate and by a weighted least-squares linear regression (the weighting factor $1 / X^{2}$ ) to obtain the calibration curve.

The lowest concentration of analyte that can be reliably quantified in the sample was the LLOQ. Six standard plasma samples containing NAR were analysed as described in Section 2.6. 
TABLE 1: Mass spectrometry parameters.

\begin{tabular}{|c|c|c|c|}
\hline Parameters & Value & Parameters & Value \\
\hline MRM ion pair of NAR & $\mathrm{m} / \mathrm{z} 271.1 \longrightarrow 151.0$ & Declustering potential (DP) & $-10 \mathrm{~V}$ \\
\hline MRM ion pair of IS & $\mathrm{m} / \mathrm{z} 301.2 \longrightarrow 164.1$ & Collision energy (CE) & $-25 \mathrm{~V}$ (NAR)-30 V (IS) \\
\hline Entrance potentials (EP) & $-10 \mathrm{~V}$ & Collision Exit Potentials (CXP) & $-8 \mathrm{~V}$ \\
\hline Temperature & $550^{\circ} \mathrm{C}$ & Ion spray voltage & $-4500 \mathrm{~V}$ \\
\hline Curtain gas & $28 \mathrm{psi}$ & Nebulizer gas & $50 \mathrm{psi}$ \\
\hline Turbo gas & 50 psi & Dwell time & $100 \mathrm{~ms}$ \\
\hline
\end{tabular}

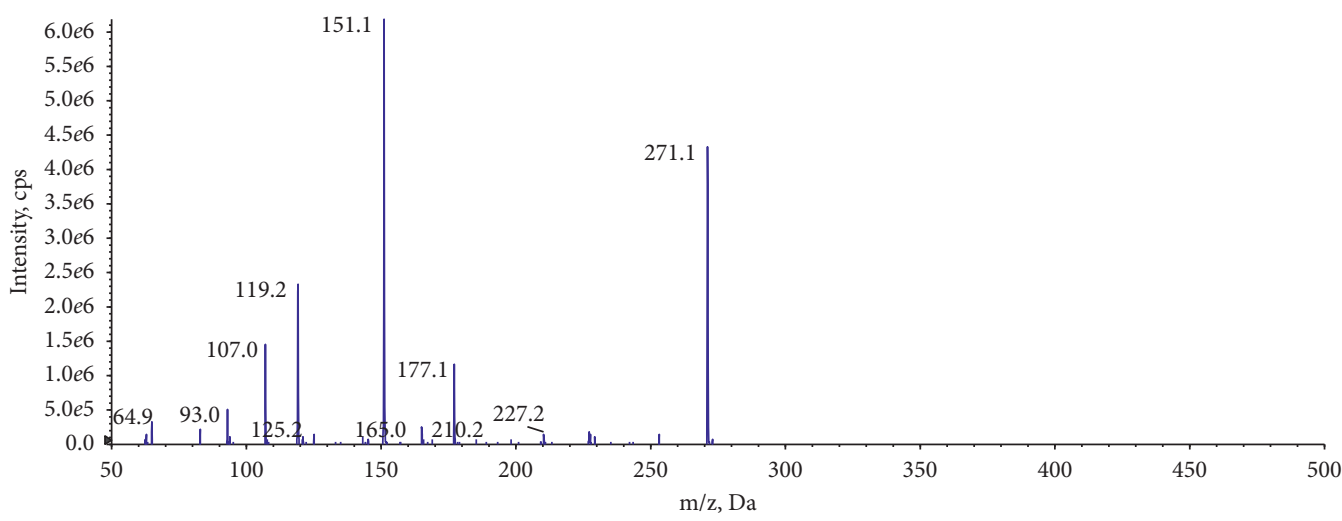

(a)

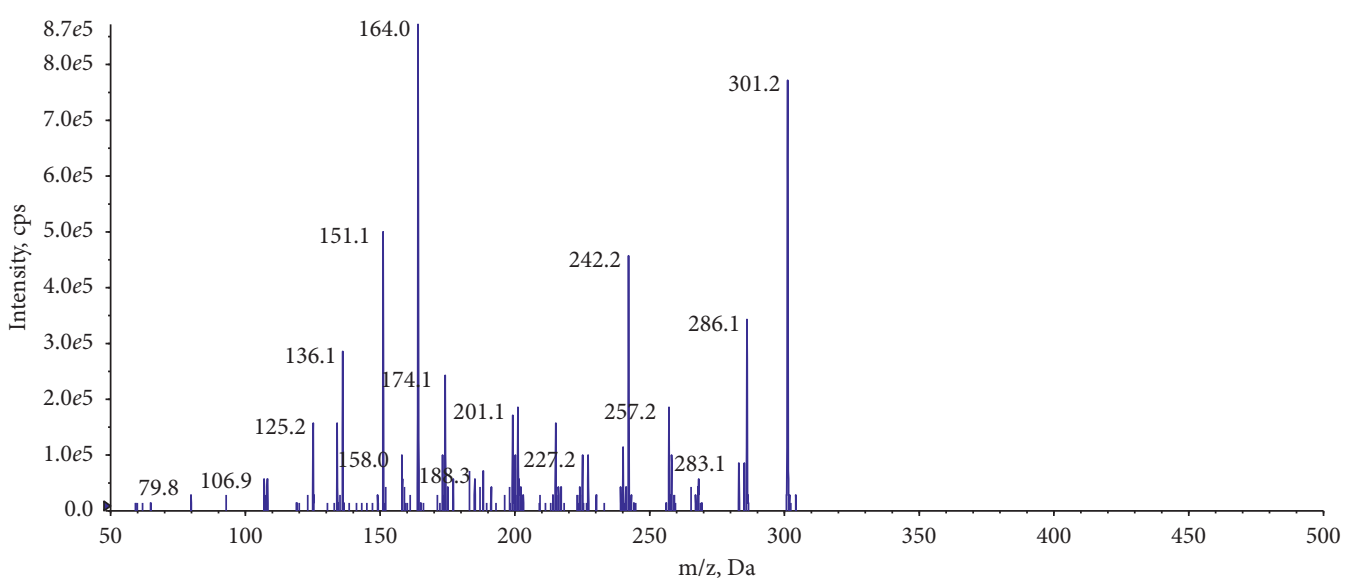

(b)

Figure 2: Mass spectrometry of the product ion: (a) NAR and (b) IS.

At the LLOQ, the relative standard deviation (RSD, \%) was $\leq 20 \%$, the relative error ( $\mathrm{RE}, \%$ ) was required to be within $\pm 20 \%$.

2.7.3. Precision and Accuracy. NAR plasma standard samples were prepared at the LLOQ, LQC, MQC, and HQC concentrations $(1.03,3.08,410$, and $616 \mathrm{ng} / \mathrm{mL})$, according to Section 2.6. Six samples were prepared at each concentration. The concentration of each sample was determined on the same day and three consecutive days to assess the intraand interday precision and accuracy.

The acceptance criteria were RSD (\%) was $\leq 15 \%$, RE (\%) was limited to $\pm 15 \%$ for the intra- and interday, except at the LLOQ, where RSD (\%) should be $\leq 20 \%$ and RE (\%) within $\pm 20 \%$.
2.7.4. Extraction Recovery (R). NAR plasma standard samples were prepared at the LLOQ, LQC, MQC, and HQC concentrations $(1.03,3.08,410$, and $616 \mathrm{ng} / \mathrm{mL})$. Then, internal standard solution $(10 \mu \mathrm{L})$ was added to the mixture, vortexed for $5 \mathrm{~min}$, and added ethyl acetate $(300 \mu \mathrm{L})$. After that, the samples were centrifuged at $8000 \mathrm{r} / \mathrm{min}$ for 2 min. All supernatant was transferred to a new tube. All of them were concentrated to dryness under flowing nitrogen, and the residue was redissolved in methanol $(200 \mu \mathrm{L})$ to prepare it for analysis. The peak area of NAR (As) was recorded.

Blank plasma samples $(100 \mu \mathrm{L})$ from different sources were mixed with ethyl acetate $(300 \mu \mathrm{L})$. The samples were vortexed for $5 \mathrm{~min}$, then centrifuged for $2 \mathrm{~min}$ at $8000 \mathrm{r} / \mathrm{min}$, and then all supernatant was removed for analysis. Aliquots 
$10 \mu \mathrm{L}$ of the standard solutions $(10.3,30.8,4104$, and $6156 \mathrm{ng} / \mathrm{mL}$ ) and $10 \mu \mathrm{L}$ of $300 \mathrm{ng} / \mathrm{mL}$ internal standard solution were added. All of them were concentrated to dryness under flowing nitrogen, and the residues were redissolved in methanol $(200 \mu \mathrm{L})$ for analysis. The peak area of NAR $\left(\mathrm{As}^{\prime}\right)$ was recorded.

The extraction recovery (\%) of NAR was obtained by substituting As and $\mathrm{As}^{\prime}$ into the following equation:

$$
R(\%)=\frac{\mathrm{As}}{\mathrm{As}^{\prime}} \times 100 \% \text {. }
$$

The recovery of the IS was investigated similarly.

2.7.5. Matrix Effect (ME). The standard solutions $(10 \mu \mathrm{L}$, at concentrations of $10.3,30.8,4104$, and $6156 \mathrm{ng} / \mathrm{mL})$ were combined with the internal standard solution $(10 \mu \mathrm{L}, 300 \mathrm{ng} /$ $\mathrm{mL}$ ) in a centrifuge tube. The mixture was vortexed (5 min), dried at $40^{\circ} \mathrm{C}$ under flowing nitrogen, and redissolved in methanol $(200 \mu \mathrm{L})$. The supernatant was then analysed. The peak area of NAR (Bs) was recorded.

Blank plasma samples $(100 \mu \mathrm{L})$, from different sources, were each mixed with ethyl acetate $(300 \mu \mathrm{L})$, and the mixture was vortexed ( $5 \mathrm{~min})$ and centrifuged $(8000 \mathrm{r} / \mathrm{min}, 2 \mathrm{~min})$. All supernatant of each sample was removed to a new tube, and standard solution $(10 \mu \mathrm{L}$, at concentrations of $10.3,30.8$, 4104, and $6156 \mathrm{ng} / \mathrm{mL}$ ) was added to the internal standard solution $(10 \mu \mathrm{L}, 300 \mathrm{ng} / \mathrm{mL})$. The sample was dried at $40^{\circ} \mathrm{C}$ under flowing nitrogen. Methanol $(200 \mu \mathrm{L})$ was used to reconstitute in the residue. The peak area of NAR $\left(\mathrm{Bs}^{\prime}\right)$ was recorded.

The matrix effect (\%) on NAR was calculated according to the following formula:

$$
\operatorname{ME}(\%)=\frac{\mathrm{Bs}^{\prime}}{\mathrm{Bs}} \times 100 \% .
$$

The ME of the IS was investigated.

2.7.6. Stability. Analyte's stability in the matrix at the LLOQ, LQC, MQC, and HQC under different conditions was investigated. The evaluated conditions were $24 \mathrm{~h}$ short-term stability at room temperature $\left(25 \pm 3^{\circ} \mathrm{C}\right)$, freeze-thaw cycles three times, long-term stability under $-80^{\circ} \mathrm{C}$ storage 21 days, and room temperature in an automatic sampler for $24 \mathrm{~h}$.

The acceptance criteria for the RSD (\%) was $\leq 15 \%$, RE (\%) was limited to $\pm 15 \%$, except at the LLOQ, where RSD(\%) was $\leq 20 \%$ and $\operatorname{RE}(\%)$ within $\pm 20 \%$.

2.7.7. Dilution Reliability. The dilution reliability of NAR in plasma must be examined because of the high concentrations (beyond the calibration curve) of some drugs in the plasma. The plasma sample (NAR, $6156 \mathrm{ng} / \mathrm{mL}$ ) was diluted with blank plasma 10 times and analysed according to Section 2.6. After sample analysis, the ratio of peak area of NAR to IS was compared to the calibration curve, and RE and RSD values were also calculated $(n=6)$. Accuracy of the concentration determined following dilution was required to be between $85 \%$ and $115 \%$, and the precision should be within $15 \%$.

2.8. Pharmacokinetic Study. Eighteen SD rats $(200 \pm 20 \mathrm{~g}, 8$ weeks, half male and half female) were obtained from $\mathrm{Na}$ tional Institutes for Food and Drug Control (Beijing, China). The room environment was about $22 \pm 2^{\circ} \mathrm{C}$, the humidity of $50 \pm 10 \%$, light and dark recycle for $12 \mathrm{~h}$. Before the experiment, the rats can be given water freely but were fasted for $12 \mathrm{~h}$. All experiments were following the National Institutes of Health Guide for the Care and Use of Laboratory Animals [24].

There were three groups for oral administration. The first group was administered NAR (30 mg/kg). The next group was administered NAR + NCT, in which the molar ratio of NAR and NCT was $1: 2$, in terms of NAR $30 \mathrm{mg} / \mathrm{kg}$. The last group was administered NAR-NCT $(57 \mathrm{mg} / \mathrm{kg})$, which was equal to the NAR dose of $30 \mathrm{mg} / \mathrm{kg}$. These were ground with $0.5 \%$ sodium carboxymethyl cellulose, at a dose of $1 \mathrm{~mL} /$ $100 \mathrm{~g}$. At $0.05,0.083,0.167,0.333,0.5,1,2,4,8,10,12$, and $24 \mathrm{~h}$ after administration, $0.5 \mathrm{~mL}$ serial blood samples were obtained from the ocular vein into centrifuge tubes. The samples were centrifuged at $10000 \mathrm{r} / \mathrm{min}\left(10 \mathrm{~min}, 4^{\circ} \mathrm{C}\right)$, and after that, all supernatant of each sample was removed to store at $-80^{\circ} \mathrm{C}$.

2.9. Data Analysis. Noncompartmental modelling was evaluated for pharmacokinetic parameters through DAS Software (China State Drug Administration, version 3.2.4).

The following equation was used to calculate the relative oral bioavailability (Fr, \%) in rats:

$$
\mathrm{Fr} \%=\frac{\mathrm{AUC}_{\mathrm{NAR}-\mathrm{NCT}} \times \text { dose }_{\mathrm{NAR}}}{\mathrm{AUC}_{\mathrm{NAR}} \times \text { dose }_{\text {NAR-NCT }}} \times 100 \% \text {. }
$$

\section{Results and Discussion}

3.1. NAR-NCT Cocrystal. The NAR-NCT cocrystal was prepared and characterized, which can improve solubility in water. The DSC endothermic peak of the NAR-NCT was $134.68^{\circ} \mathrm{C}$. The characteristic peaks in the DSC, XRPD, and IR spectra of NAR-NCT were different from the naringeninnicotinamide cocrystal reported in the literature [25]. It is shown that a new NAR-NCT cocrystal was formed. The DSC, XRPD, and IR spectra are shown in Figure 3.

3.2. Stability of NAR-NCT. The stability of NAR-NCT was investigated at $60^{\circ} \mathrm{C}$, at a relative humidity of $90 \pm 5 \%$, under light of $5000 \mathrm{Lx}$ for 10 days and after 6 months at room temperature. In terms of appearance, NAR-NCT showed no difference under the above conditions. A comparison of the XRPD spectra (Figure 4) showed that the overall peak shape, position, and intensity are not a significant difference, indicating that the NAR-NCT was stable at $60^{\circ} \mathrm{C}$, at a relative humidity of $90 \pm 5 \%$, under light of $5000 \mathrm{Lx}$ for 10 days and after 6 months at room temperature. 


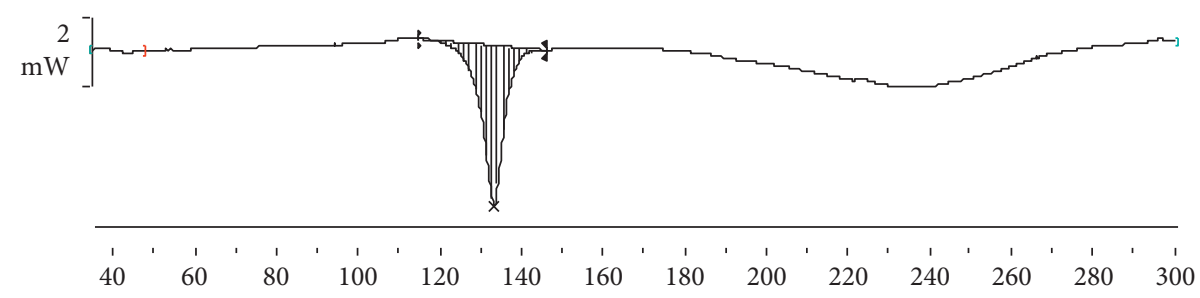

(a)

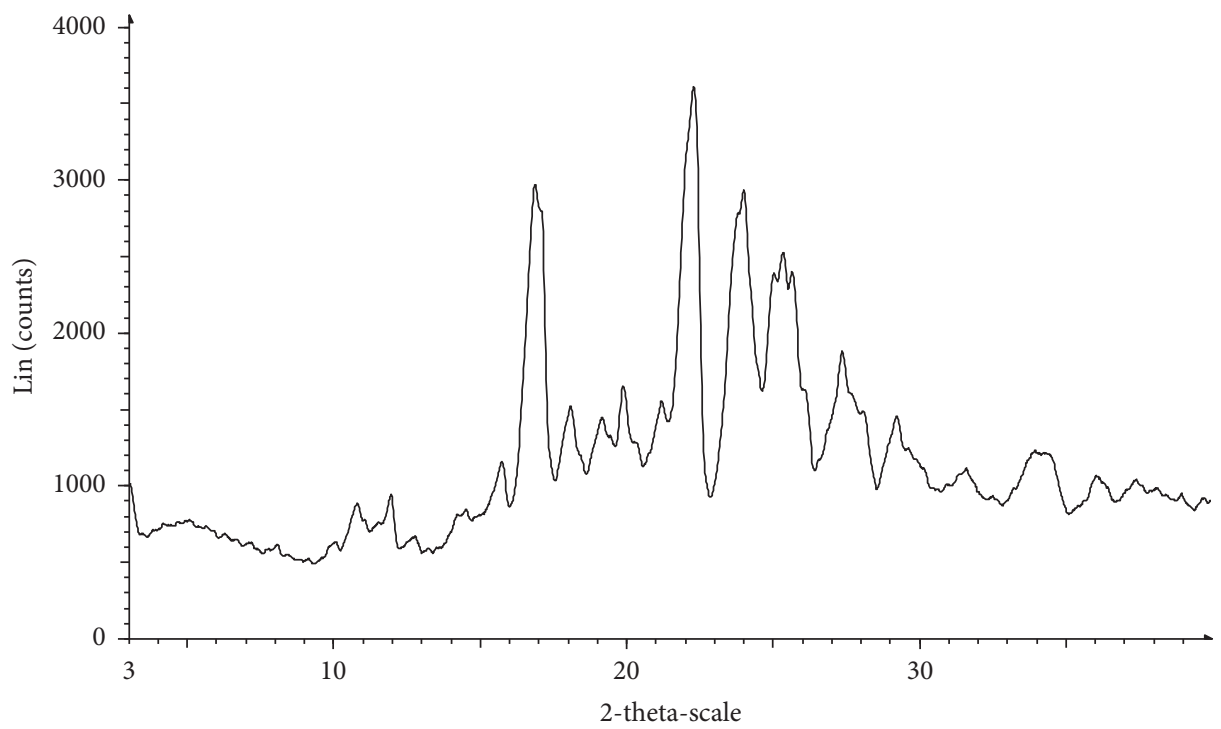

(b)

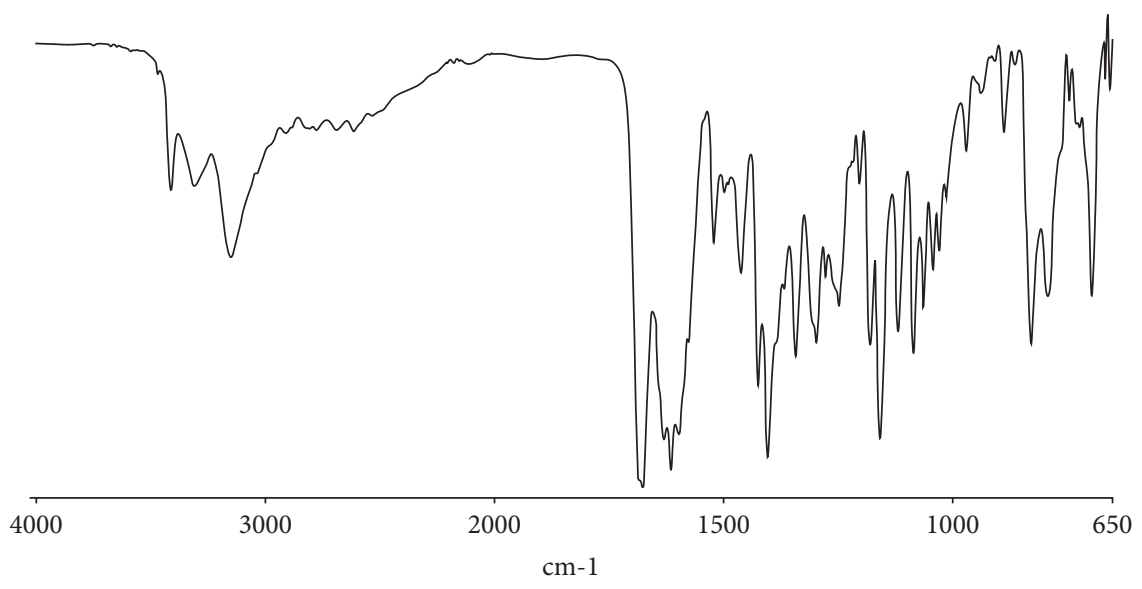

(c)

FIgURE 3: The spectrum of NAR-NCT: (a) DSC, (b) XRPD, and (c) IR.

3.3. Optimization of the Plasma Sample Preparation. Protein precipitation and liquid-liquid extraction methods were tested for the removal of interfering species from the sample. Organic solvents, such as methanol, acetonitrile, diethyl ether, ethyl acetate, and n-hexane were investigated, and the results are shown in Figure 5. The extraction effect of ethyl acetate was the best, so the extraction effects of 100 , 200,300 , and $400 \mu \mathrm{L}$ of ethyl acetate were analysed. Results showed that ethyl acetate $(300 \mu \mathrm{L})$ had the best extraction effect. Therefore, ethyl acetate $(300 \mu \mathrm{L})$ was used to the extracted solvent for pretreatment.
3.4. Method Validation. The blank plasma sample showed no signals interfering with the measurement of the analyte. NAR and IS were well separated from endogenous substances and metabolites, and no interference was observed. The chromatograms are shown in Figure 6.

The calibration curve showed good linearity, at the range of $1.03-821 \mathrm{ng} / \mathrm{mL}, Y=0.11 X+0.0914$, and $r=0.9995$. The standard error of slope and intercept was 0.002 and 0.005 , respectively. LLOQ was $1.03 \mathrm{ng} / \mathrm{mL}$ for the NAR plasma sample.

The results of precision and accuracy during intraday and interday were as follows: both RSD (\%) values were 


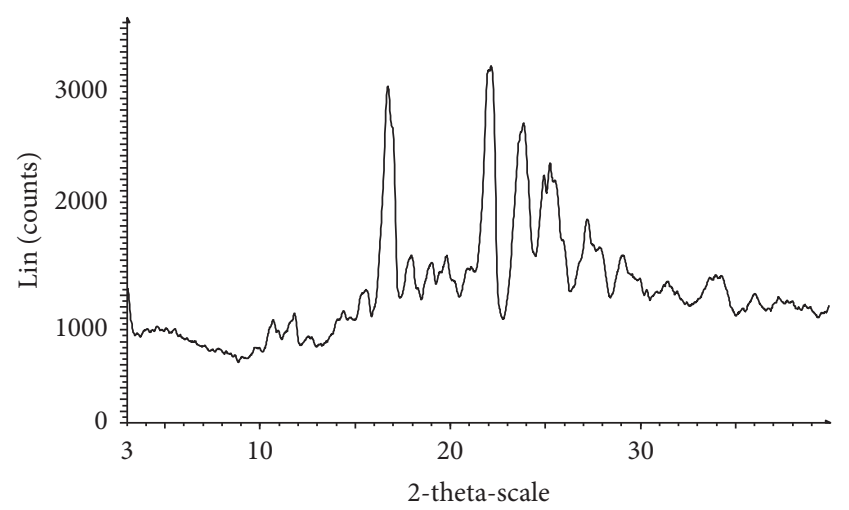

(a)

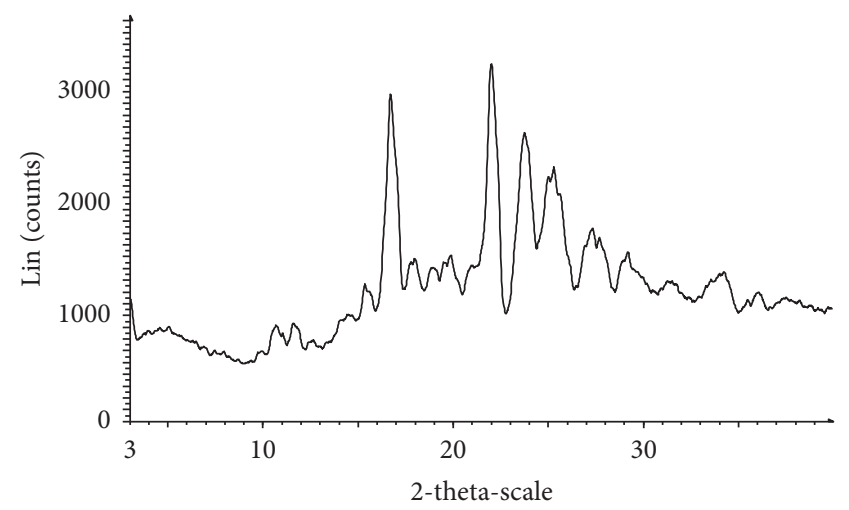

(c)

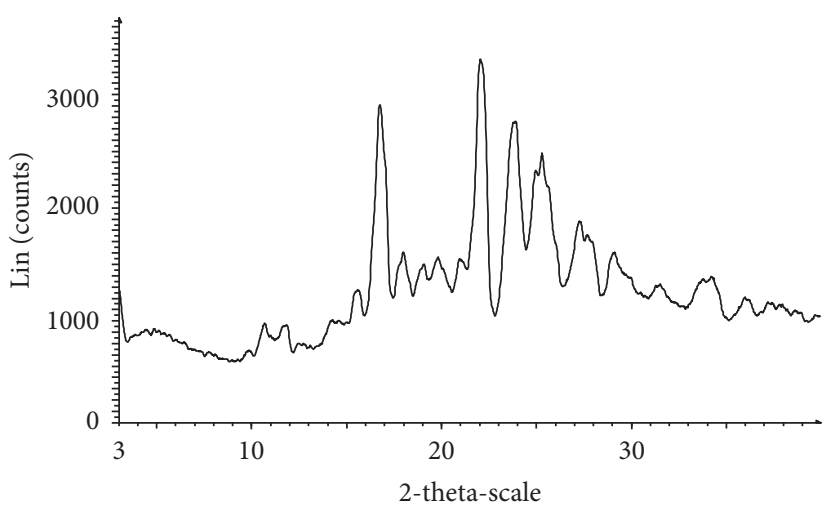

(b)

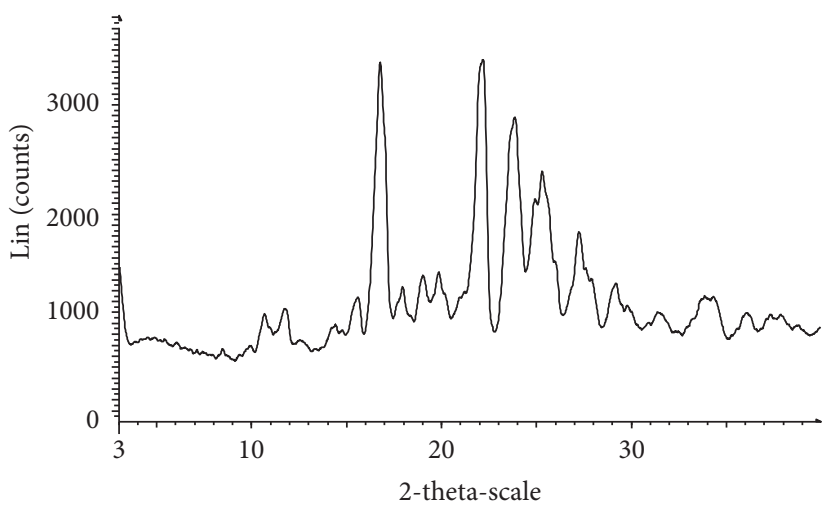

(d)

Figure 4: The XRPD pattern of NAR-NCT (a) $60^{\circ} \mathrm{C}$ for 10 days, (b) relative humidity of $90 \pm 5 \%$ for 10 days, and (c) light of $5000 \mathrm{Lx}$ for 10 days, (d) 6 months at room temperature.

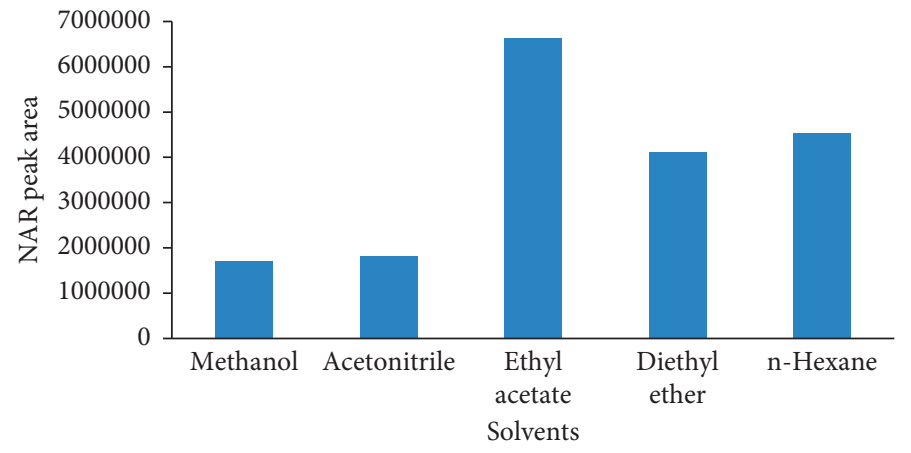

(a)

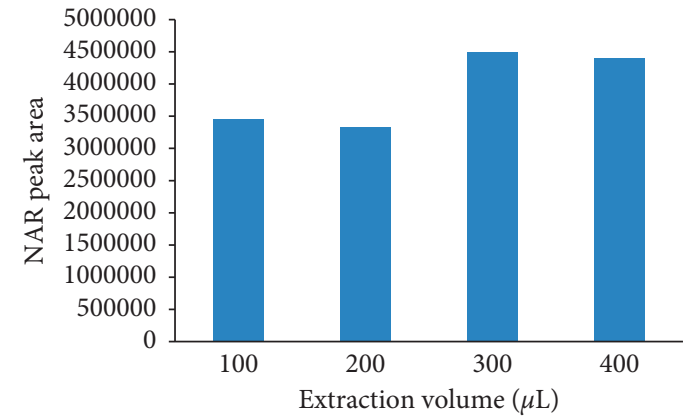

(b)

FIgURE 5: Effects of solvents on NAR extraction: (a) solvent selection and (b) ethyl acetate volume.

$\leq 8.5 \%$, while both $\mathrm{RE}(\%)$ values were at the range of $-6.2 \%$ to $4.7 \%$ and $-2.6 \%$ to $2.8 \%$, respectively (Table 2 ).

The extraction recoveries were $69.0 \%$ to $76.5 \%$, and RSD (\%) values were $\leq 10.2 \%$. The matrix effects were $107.7 \%$ to $131.2 \%$, and RSD (\%) values were $\leq 7.7 \%$ (Table 2 ).

The plasma samples were stable for $24 \mathrm{~h}$ at room temperature $\left(25 \pm 3^{\circ} \mathrm{C}\right)$ with the $\mathrm{RE} \%$ value of $0.9 \%$ to $10.3 \%$. After three freeze-thaw cycles, NAR also remained stable
(RE\%: $1.6 \%$ to $4.8 \%$ ). The sample exhibits 21 days of longterm stability (RE\%: $-6.9 \%$ to $0.2 \%$ ) at $-80^{\circ} \mathrm{C}$, and the readyto-inject sample was stable for $24 \mathrm{~h}$ in an autosampler at $25^{\circ} \mathrm{C}$ (RE\%: $-8.4 \%$ to $4.4 \%$ ). These results are recorded in Table 2 .

After the plasma QC samples were diluted with blank plasma, the RE ranged from $-8.1 \% \%$ to $1.9 \%$, and the RSD was $4.7 \%$ (Table 3 ). These results met the requirements of methodological validation. 

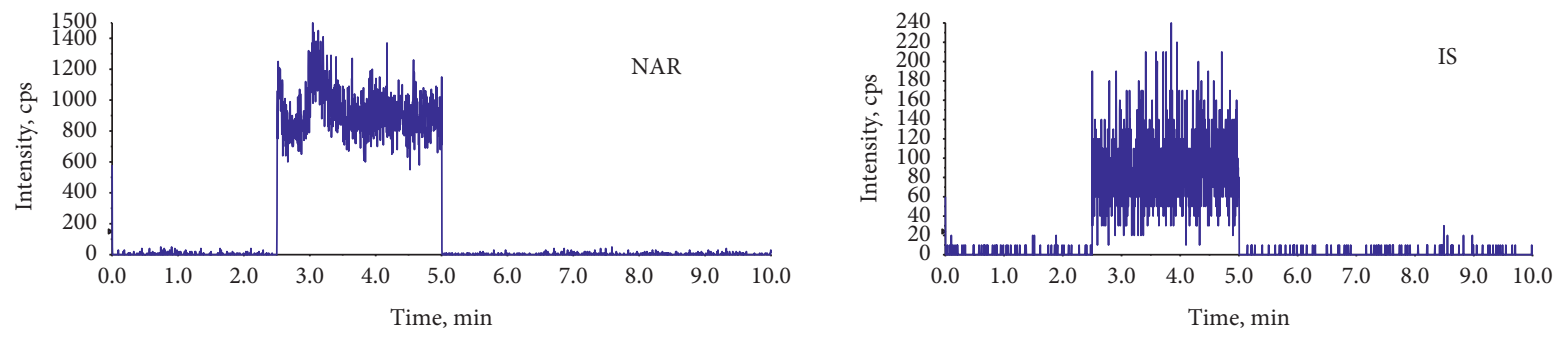

(a)
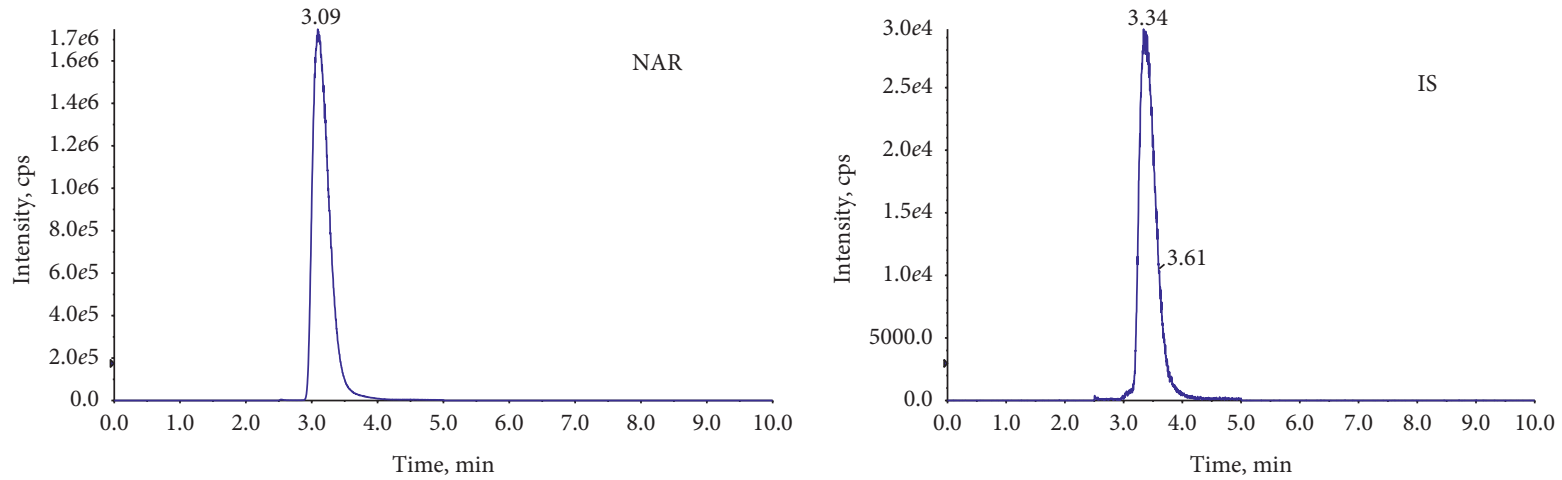

(b)
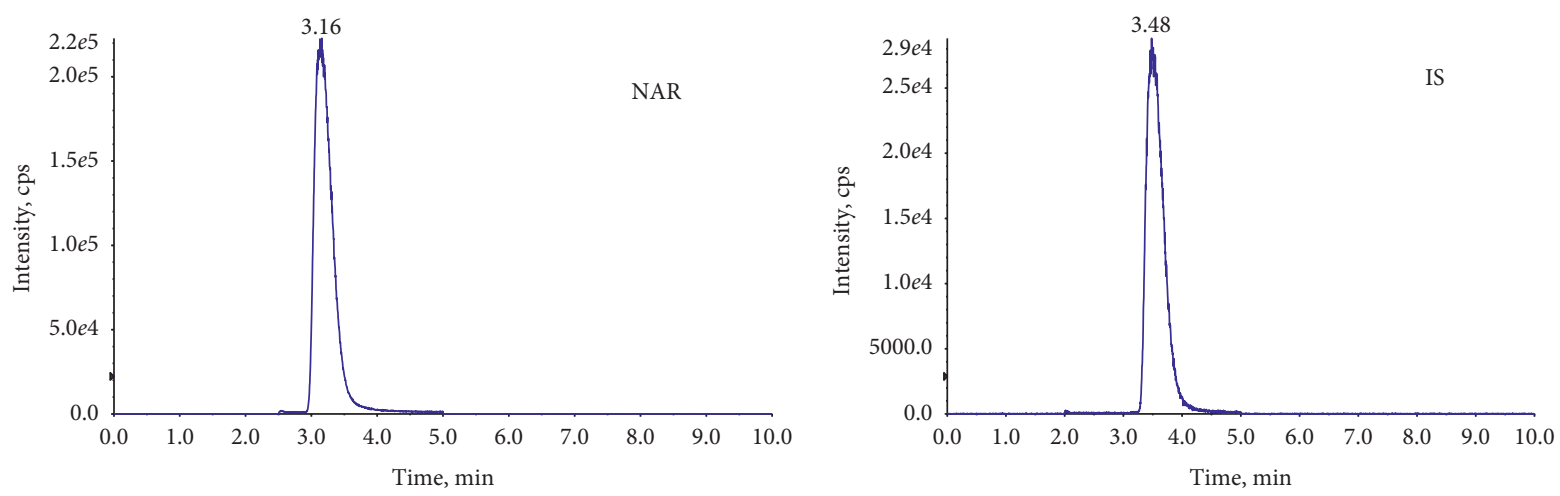

(c)

FIgURE 6: The chromatograms of MRM: (a) blank plasma, (b) plasma mixed with NAR, and (c) plasma after oral administration of NAR.

3.5. Pharmacokinetics and Bioavailability. The plasma concentrations of NAR were determined by LC-MS/MS. The concentrations were analysed with DAS Software. The main pharmacokinetic parameters are recorded in Table 4, and the mean plasma concentration versus time curves for NAR is shown in Figure 7.

Compared with NAR, the $\mathrm{C}_{\max }$ of NAR + NCT was greater by a factor of 2.06 , and $\mathrm{T}_{\max }$ changed from $0.49 \mathrm{~h}$ to $0.11 \mathrm{~h}$. Although NAR + NCT accelerated drug absorption, it did not improve drug metabolism. The values of CL and $t_{1 / 2}$ showed that the elimination rates of the two groups were similar. As a result, the relative bioavailability was $77.23 \%$ of NAR.

A comparison between NAR-NCT and NAR showed that $\mathrm{C}_{\max }$ was increased by a factor of 8.43 , and $\mathrm{T}_{\max }$ was advanced from $0.49 \mathrm{~h}$ to $0.09 \mathrm{~h}$. In contrast to NAR + NCT, the CL of NAR-NCT decreased from $91.1 \mathrm{~L} / \mathrm{h} / \mathrm{kg}$ to $49.1 \mathrm{~L} / \mathrm{h} / \mathrm{kg}$, and $t_{1 / 2}$ increased from $5.37 \mathrm{~h}$ to $8.24 \mathrm{~h}$, showing the rapid absorption and slow elimination of this formulation. The relative bioavailability of NAR-NCT was $175.09 \%$ of NAR, compared with $80.89 \%$ reported in the literature [25]. This result showed that NAR-NCT can improve bioavailability and has the advantages of fast absorption and slow elimination. The above phenomenon conformed to the hypothesis [26]. NCT was easily soluble in water, and in aqueous biological environments, NCT was more easily detached from the crystal lattice and readily entered into solution. Then, the NAR particles remained in the solution medium. Because the rate of NAR-NCT dissolution was faster than the formation of supramolecular aggregates of the NAR molecule itself, $\mathrm{C}_{\max }$ increased and $\mathrm{T}_{\max }$ decreased. After the dissolution of NCT, the amorphous NAR, with high free energy, slowly converted to a metastable state and then to the lowest energy NAR configuration (usually the pure drug). The time required for this process resulted in slower NAR elimination and increased AUC in the body.

The peak time of NAR, NAR + NCT, and NAR-NCT is $0.49 \mathrm{~h}, 0.11 \mathrm{~h}$, and $0.09 \mathrm{~h}$, respectively, so NAR has reached the 
TABLE 2: Method validation of NAR in rat plasma $(n=6)$.

\begin{tabular}{|c|c|c|c|c|c|c|}
\hline & & & \multicolumn{4}{|c|}{ NAR concentration $(\mathrm{ng} / \mathrm{mL})$} \\
\hline & & & 1.03 & 3.08 & 410 & 616 \\
\hline \multirow{4}{*}{ Precision accuracy } & \multirow{2}{*}{ Intraday } & $\mathrm{RE}(\%)$ & 1.4 & -6.2 & 4.7 & 3.4 \\
\hline & & RSD (\%) & 8.3 & 8.5 & 3.2 & 4.3 \\
\hline & \multirow{2}{*}{ Interday } & RE (\%) & 2.8 & -2.6 & -0.5 & -1.4 \\
\hline & & RSD (\%) & 1.7 & 6.2 & 6.9 & 6.3 \\
\hline \multirow{2}{*}{ Recovery } & & $\mathrm{R}(\%)$ & 69.0 & 69.5 & 69.9 & 76.5 \\
\hline & & RSD (\%) & 9.0 & 6.5 & 3.7 & 10.2 \\
\hline \multirow{2}{*}{ Matrix effect } & & ME (\%) & 111.4 & 131.2 & 116.9 & 107.7 \\
\hline & & RSD (\%) & 7.7 & 5.3 & 2.6 & 1.1 \\
\hline \multirow{8}{*}{ Stability } & \multirow{2}{*}{ Short-term $(24 \mathrm{~h})$} & $\mathrm{RE}(\%)$ & 3.5 & 10.3 & 0.9 & 1.7 \\
\hline & & RSD (\%) & 6.9 & 3.2 & 7.8 & 0.5 \\
\hline & \multirow{2}{*}{ Freeze-thaw cycles } & $\mathrm{RE}(\%)$ & 1.8 & 2.8 & 4.8 & 1.6 \\
\hline & & RSD (\%) & 11.1 & 12.2 & 3.9 & 2.3 \\
\hline & \multirow{2}{*}{ Long-term (21 days) } & RE (\%) & 0.2 & -6.5 & 0.1 & -6.9 \\
\hline & & RSD (\%) & 6.2 & 10.1 & 5.1 & 2.1 \\
\hline & \multirow{2}{*}{ Autosampler (24 h) } & RE (\%) & 2.0 & 4.4 & -8.4 & -8.3 \\
\hline & & RSD (\%) & 5.4 & 9.3 & 3.6 & 4.0 \\
\hline
\end{tabular}

TABLE 3: Dilution reliability of NAR plasma samples $(n=6)$.

\begin{tabular}{|c|c|c|c|c|c|}
\hline Compound & Nominal concentration $(\mathrm{ng} / \mathrm{mL})$ & Dilution factor & Measured concentration $(\mathrm{ng} / \mathrm{mL})$ & $\mathrm{RE}(\%)$ & RSD (\%) \\
\hline \multirow{6}{*}{ NAR } & \multirow{6}{*}{6156} & \multirow{6}{*}{10} & 599 & -2.8 & \multirow{6}{*}{4.7} \\
\hline & & & 566 & -8.1 & \\
\hline & & & 628 & 1.9 & \\
\hline & & & 573 & -7 & \\
\hline & & & 626 & 1.6 & \\
\hline & & & 571 & -7.3 & \\
\hline
\end{tabular}

TABLE 4: Noncompartmental pharmacokinetic parameters of NAR after oral administration.

\begin{tabular}{lccc}
\hline & NAR & NAR + NCT & NAR-NCT \\
\hline$T_{\text {max }}(\mathrm{h})$ & $0.49 \pm 0.7$ & $0.11 \pm 0.05^{* * * *}$ & $0.09 \pm 0.02^{* * * *}$ \\
$C_{\text {max }}(\mathrm{ng} / \mathrm{mL})$ & $120.8 \pm 95.0$ & $494.0 \pm 427.4^{* *}$ & $1018.3 \pm 853.4^{* * *}$ \\
$t_{1 / 2}(\mathrm{~h})$ & $5.37 \pm 2.54$ & $5.50 \pm 2.44$ & $8.24 \pm 6.88^{*}$ \\
$\mathrm{CL} / \mathrm{F}(\mathrm{L} / \mathrm{h} / \mathrm{kg})$ & $91.1 \pm 58.0$ & $86.4 \pm 48.0$ & $49.1 \pm 22.5$ \\
$\mathrm{~V} / \mathrm{F}(\mathrm{L} / \mathrm{kg})$ & $575.1 \pm 259.6$ & $580.8 \pm 165.0$ & $670.5 \pm 766.8^{*}$ \\
$\mathrm{MRT}_{(0-t)}(\mathrm{h})$ & $5.4 \pm 0.9$ & $4.2 \pm 1.0$ & $3.9 \pm 1.9$ \\
$\mathrm{MRT}_{(0-\infty)}(\mathrm{h})$ & $6.8 \pm 2.5$ & $5.3 \pm 2.2$ & $8.0 \pm 9.1^{*}$ \\
$\mathrm{AUC}_{0-t}(\mathrm{ng} /$ & $509.4 \pm 485.2$ & $393.4 \pm 135.6^{*}$ & $891.9 \pm 963.0$ \\
$\left.\mathrm{~mL}^{*} \mathrm{~h}\right)$ & & & \\
$\mathrm{AUC}_{0-\infty}(\mathrm{ng} /$ & $567.6 \pm 604.0$ & $413.8 \pm 156.6^{*}$ & $936.4 \pm 943.1$ \\
$\left.\mathrm{~mL}^{*} \mathrm{~h}\right)$ & & & \\
$\mathrm{Fr}$ & & 77.23 & 175.09 \\
\hline
\end{tabular}

${ }^{* * * *} P<0.0001,{ }^{* * *} P<0.001,{ }^{* *} P<0.01$, and ${ }^{*} P<0.05$.

peak at $0.5 \mathrm{~h}$. And the last points of the pharmacokinetic study in the three groups were less than $1 / 10$ of $C_{\max }$, respectively. After $0.5 \mathrm{~h}$, a longer interval of blood collection was set. The rats could be allowed to drink water freely in longer blood sampling intervals. Therefore, the process of blood sampling did not have a serious impact on the pharmacokinetics of NAR.

Only the drug-time curve of NAR-NCT showed the double peaks, which was consistent with enterohepatic

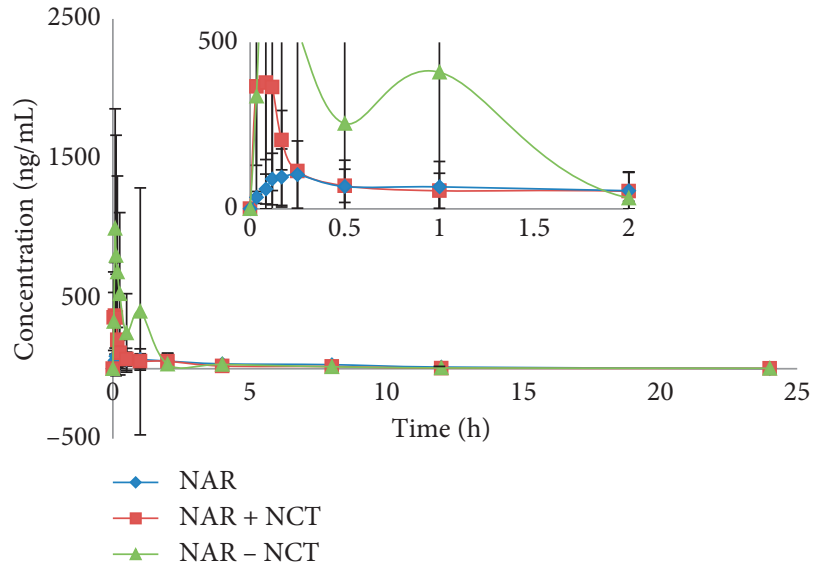

FIGURE 7: Drug concentration-time curve (in terms of NAR $30 \mathrm{mg}$ / $\mathrm{kg})(n=6)$.

circulation [27] and extended NAR action time in the body.

\section{Conclusions}

We investigated the differences in pharmacokinetic characteristics and the relative bioavailability of NAR after oral 
administration of NAR, NAR + NCT, and NAR-NCT in rats. These results show that NAR-NCT was a viable method for improving the bioavailability of NAR. These results provide important references for the application and research of NAR in the future.

\section{Data Availability}

All data included in this study are available upon request from the corresponding author.

\section{Conflicts of Interest}

The authors declare they have no conflicts of interest.

\section{Acknowledgments}

This work was supported by the National Major Scientific and Technological Special Project for "Significant New Drugs Development” (2012ZX09301002-001-006).

\section{References}

[1] A. H. Hegde, S. N. Prashanth, and J. Seetharamappa, "Interaction of antioxidant flavonoids with calf thymus DNA analyzed by spectroscopic and electrochemical methods," Journal of Pharmaceutical and Biomedical Analysis, vol. 63, pp. 40-46, 2012.

[2] W. Wojnar, M. Zych, and I. Kaczmarczyk-Sedlak, "Antioxidative effect of flavonoid naringenin in the lenses of type 1 diabetic rats," Biomedicine \& Pharmacotherapy, vol. 108, pp. 974-984, 2018.

[3] Y. Bak, H. Kim, J.-W. Kang et al., "A synthetic naringenin derivative, 5-Hydroxy-7,4'-diacetyloxyflavanone-N-phenyl hydrazone (N101-43), induces apoptosis through up-regulation of fas/FasL expression and inhibition of PI3K/akt signaling pathways in non-small-cell lung cancer cells," Journal of Agricultural and Food Chemistry, vol. 59, no. 18, pp. 10286-10297, 2011.

[4] H. Gumushan and T. Akgun, "Naringenin inhibits prostate cancer metastasis by blocking voltage-gated sodium channels," Biopha, vol. 106, pp. 770-775, 2018.

[5] S.-J. Tsai, C.-S. Huang, M.-C. Mong, W.-Y. Kam, H.-Y. Huang, and M.-C. Yin, "Anti-inflammatory and antifibrotic effects of naringenin in diabetic mice," Journal of Agricultural and Food Chemistry, vol. 60, no. 1, pp. 514-521, 2012.

[6] M. A. Alam, N. Subhan, M. M. Rahman, S. J. Uddin, H. M. Reza, and S. D. Sarker, "Effect of citrus flavonoids, naringin and naringenin, on metabolic syndrome and their mechanisms of action," Advances in Nutrition, vol. 5, no. 4, pp. 404-417, 2014.

[7] S. Kumar and A. B. Tiku, "Biochemical and molecular mechanisms of radioprotective effects of naringenin, a phytochemical from citrus fruits," Journal of Agricultural and Food Chemistry, vol. 64, no. 8, pp. 1676-1685, 2016.

[8] C. Zhang, W. Zeng, Y. Yao et al., "Naringenin ameliorates radiation-induced lung injury by lowering IL-1 $\beta$ level," Journal of Pharmacology and Experimental Therapeutics, vol. 366, no. 2, pp. 341-348, 2018.

[9] W. F. Zeng, F. Y. Zhang, G. J. Du et al., "Research updates on a novel immunomodulator, naringenin," Progress in Biochemistry and Biophysics, vol. 45, pp. 915-925, 2018.
[10] B.-q. Lin, P.-b. Li, Y.-g. Wang et al., "The expectorant activity of naringenin," Pulmonary Pharmacology \& Therapeutics, vol. 21, no. 2, pp. 259-263, 2008.

[11] J. Wen, Y. Qiao, J. Yang et al., "UPLC-MS/MS determination of paeoniflorin, naringin, naringenin and glycyrrhetinic acid in rat plasma and its application to a pharmacokinetic study after oral administration of SiNiSan decoction," Journal of Pharmaceutical and Biomedical Analysis, vol. 66, pp. 271-277, 2012.

[12] T. T. Zhang and C. Ma, "Determination of naringenin and its glucuronic acid conjugate in rat plasma by LC-MS/MS," Journal of Chinese Pharmaceutical Sciences, vol. 49, no. 10, pp. 864-868, 2014.

[13] S. Chaurasia, R. R. Patel, P. Vure, and B. Mishra, "Oral naringenin nanocarriers: fabrication, optimization, pharmacokinetic and chemotherapeutic efficacy assessments," Nanomedicine, vol. 12, no. 11, pp. 1243-1260, 2017.

[14] S. L. Hsiu, T. Y. Huang, Y. C. Hou, D. H. Chin, and P. D. Lee Chao, "Comparison of metabolic pharmacokinetics of naringin and naringenin in rabbits," Life Sciences, vol. 70, no. 13, pp. 1481-1489, 2002.

[15] C. Yang, W. Guo, Y. Lin et al., "Experimental and DFT simulation study of a novel felodipine cocrystal: characterization, dissolving properties and thermal decomposition kinetics," Journal of Pharmaceutical and Biomedical Analysis, vol. 154, pp. 198-206, 2018.

[16] N. Wang, C. Xie, H. Lu et al., "Cocrystal and its application in the field of active pharmaceutical ingredients and food ingredients," Current Pharmaceutical Design, vol. 24, no. 21, pp. 2339-2348, 2018.

[17] A. Kozak, P. H. Marek, and E. Pindelska, "Structural characterization and pharmaceutical properties of three novel cocrystals of ethenzamide with aliphatic dicarboxylic acids," Journal of Pharmaceutical Sciences, vol. 108, no. 4, pp. 1476-1485, 2019.

[18] B. Park, W. Yoon, J. Yun, E. Ban, H. Yun, and A. Kim, "Emodin-nicotinamide $(1: 2)$ cocrystal identified by thermal screening to improve emodin solubility," International Journal of Pharmaceutics, vol. 557, pp. 26-35, 2019.

[19] W. Li, J. Pi, Y. Zhang et al., "A strategy to improve the oral availability of baicalein: the baicalein-theophylline cocrystal," Fitoterapia, vol. 129, pp. 85-93, 2018.

[20] M. Liu, C. Hong, Y. Yao et al., "Development of a pharmaceutical cocrystal with solution crystallization technology: preparation, characterization, and evaluation of myricetinproline cocrystals," European Journal of Pharmaceutics and Biopharmaceutics, vol. 107, pp. 151-159, 2016.

[21] D. P. McNamara, S. L. Childs, J. Giordano et al., "Use of a glutaric acid cocrystal to improve oral bioavailability of a low solubility API," Pharmaceutical Research, vol. 23, no. 8, pp. 1888-1897, 2006.

[22] D. Xu, T. Li, T. T. Zhang et al., "Preparation and characterization of naringin nicotinamide cocrystal," Chinese Pharmaceutical Journal, vol. 54, pp. 291-296, 2019.

[23] Center for Drug Evaluation and Research (CDER), Center of Veterinary Medicine (CVM), Guidance for Industry: Bioanalytical Method Validation, F.D.A. U.S. Department of Health and Human Services, Silver Spring, MD, USA, 2013.

[24] Institute of Laboratory Animal Resources, Guide for the Care and Use of Laboratory Animals, National Research Council, Washington, DC, USA, 2011.

[25] W. X. Cui, Z. H. He, Y. T. Zhang et al., "Naringenin cocrystals prepared by solution crystallization method for improving 
bioavailability and anti-hyperlipidemia effects," AAPS PharmSciTech, vol. 20, p. 115, 2019.

[26] G. Bolla and G. Bolla, "Pharmaceutical cocrystals: walking the talk," Chemical Communications, vol. 52, no. 54, pp. 83428360, 2016.

[27] Y. Ma, P. Li, D. Chen, T. Fang, H. Li, and W. Su, "LC/MS/MS quantitation assay for pharmacokinetics of naringenin and double peaks phenomenon in rats plasma," International Journal of Pharmaceutics, vol. 307, no. 2, pp. 292-299, 2006. 